-Original-

\title{
Ligating LAD with Its Whole Length Rather than Diagonal Branches as Coordinates Is More Advisable in Establishing Stable Myocardial Infarction Model of Swine
}

\author{
Zheyong $\mathrm{HUANG}^{1)}$, Junbo GE ${ }^{1,2)}$, Aijun $\mathrm{SUN}^{1)}$, Yanyan $\mathrm{WANG}^{1)}$, Shaoheng ZHANG ${ }^{1)}$, \\ Jie CUI ${ }^{1)}$, Suning $\mathrm{ZHANG}^{1)}$, Juying QIAN ${ }^{1)}$, and Yunzeng $\mathrm{ZOU}^{1,2)}$ \\ ${ }^{1)}$ Shanghai Institute of Cardiovascular Diseases, Zhongshan Hospital, Fudan University, Shanghai \\ 200032, China and ${ }^{2)}$ Institutes of Biomedical Science, Fudan University, Shanghai 200032, China
}

\begin{abstract}
A reproducible and reliable myocardial infarction (MI) model with less inter-individual variation in ischemic size and ventricular function is essential in cardiovascular research. Little is known about whether the different ligation coordinates [whole length of left anterior descending artery (LAD) or diagonal branches] affect the inter-individual variation of ventricular function in the MI model. The present study compared the characteristics of the experimental swine $M I$ model induced by surgical occlusion of LAD in two groups: group $A(n=24)$, where ligation was performed below the second ventricular branch $\left(D_{2}\right.$ branch), and group $B(n=23)$, where ligation was performed at a distance one-third distal to the apex. Variation of ischemic size and left ventricular ejection fraction (LVEF) at 4 weeks after MI was compared between the two groups using the homoscedasticity $F$ test and coefficient of variance $(C V)$. Difficulty in identifying ventricular branches and the great variation of branching patterns encumbered the precise ligation of LAD in group $A$. The ischemic size and LVEF in group $B$ were less variable than those of group $A$. There were significant correlations between the percentile of LAD ligation and infarct size or ventricular function. In conclusion, ligating LAD using its whole length rather than ventricular branches as coordinates may be more practical and advisable for establishing reproducible $\mathrm{MI}$ models, and this procedure may prove to help standardize the location of occlusion and infarct size.
\end{abstract}

Key words: cardiac function, coronary vessels, disease models, myocardial infarction

\section{Introduction}

There are certain anatomical and physiological similarities between pigs and humans, which makes the pig an ideal model for the study of ischemic heart disease $[8,14,19]$. Ligation of the left anterior descending artery (LAD) has been commonly used for establishing a myo- cardial infarction (MI) model due to its simplicity and convenience. Various locations of occlusion with different ligation coordinates (either the whole length of LAD or diagonal branches) have been reported, varying from occluding LAD distal to the first $\left(\mathrm{D}_{1}\right)$ [2, 12, 13], second $\left(D_{2}\right)[3,11]$, or third $\left(D_{3}\right)[16]$ ventricular branch, at the mid-point [7], to one-third [4-6] of the LAD dis-

(Received 2 September 2009 / Accepted 8 January 2010)

Address corresponding: J. Ge, Shanghai Institute of Cardiovascular Diseases, Zhongshan Hospital, Fudan University, 180 Fenglin Road, Shanghai 200032, China 
tance from the apex. A reproducible and reliable MI model with less variation in the ischemic size and ventricular function is essential in cardiovascular research. Although the inverse relationship between the ligation level and left ventricular ejection fraction (LVEF) has been established for decades in both animal and clinical studies, little is known about whether different ligation coordinates (the whole length of LAD, or diagonal branches) affect the stability of the MI model. In the present study, we compared the characteristics of an experimental MI model in miniature pigs by ligating LAD with two different coordinates: ligating LAD distal to the $\mathrm{D}_{2}$ branch (using diagonal branches for reference), and ligating LAD at a distance one-third distal to the apex (using the whole length of LAD as the reference).

\section{Materials and Methods}

\section{Animals and groups}

Forty-seven miniature pigs aged 8-11 months and weighing 17-22 kg (Huaxin Special Breeding Farm in Laogang Town, Nanhui District, Shanghai, China; Licence No. SCXK-Shanghai 2007-0013) were assigned to two groups. In group A ( $\mathrm{n}=24$, MW $18.5 \pm 3.6 \mathrm{~kg}$ ), LAD was ligated just below the $\mathrm{D}_{2}$ branch through intercostal space thoracotomy, and in group $B(n=23, M W$ $19.1 \pm 2.8 \mathrm{~kg}$ ), LAD was ligated at one-third of the distance from the apex using parasternal thoracotomy. Experiments were performed using both male and female pigs at random. All animal experiments were approved by the Animal Care and Use Committee of Fudan University (Shanghai, China) in compliance with the "Guide for the Care and Use of Laboratory Animals" published by the National Academy Press (NIH Publication No. 85-23, revised 1996).

\section{Surgical procedures}

The miniature pigs were fasted for $6 \mathrm{~h}$ and sedated with ketamine $(15-20 \mathrm{mg} / \mathrm{kg})$, diazepam $(1.5-2 \mathrm{mg} / \mathrm{kg})$, and atropine $(50-100 \mu \mathrm{g} / \mathrm{kg})$. The anesthesia was maintained with pentobarbital sodium $(0.1-0.2 \mathrm{mg} / \mathrm{kg} / \mathrm{min}$, i.v.), and succinylcholine ( $10 \mathrm{mg} / \mathrm{kg}$ ) was added to ensure thorough muscular relaxation when necessary. All animals were intubated and mechanically ventilated with oxygen and underwent electrocardio-monitoring prior to and during surgery.

The animals in group A were fixed in a right lateral position and subjected to thoracotomy at approximately the third or fourth left intercostal space where the apical beat was most palpable. After opening the left thoracic cavity, the pericardium was incised along the longitudinal axis of the heart. A silk suture was placed around the LAD approximately $3 \mathrm{~mm}$ below the $\mathrm{D}_{2}$ branch to allow partial occlusion for 15-20 min and then to ligate the vessel totally and permanently. Occlusion was confirmed by electrocardiography (elevation of the T-wave as the first sign, and later significant elevation or depression of the ST segment), gross appearance of the local myocardium (color change from pink to dark red, along with hypokinesis), and echocardiography (apical hypokinesis) $60 \mathrm{~min}$ after ligation. A lidocaine bolus $(2 \mathrm{mg} /$ $\mathrm{kg}$ ) before coronary occlusion and a subsequent infusion at a rate of $50 \mu \mathrm{g} \cdot \mathrm{kg}^{-1} \cdot \mathrm{min}^{-1}$ after occlusion were given intravenously. After stabilization of electrocardiograms and vital signs for $30 \mathrm{~min}$, the pericardium was sutured, and the wound was closed in layers, then the thoracic cavity was evacuated. The animals were allowed to recover and fed a normal diet.

The animals in group B were placed in a supine position for a left parasternal thoracotomy, approximately at the joints of the sternum and the costal cartilage. With the pleura kept intact, the pericardium was opened and LAD was occluded at one-third of the distance from the apex (positioned by trisection of a suture silk expanding from the LAD ostium to the apex). The other procedures were much the same as for group A, except that thoracic evacuation was not performed.

\section{Echocardiography and coronary angiography}

Echocardiography was performed before infarction and $1 \mathrm{~h}$ after infarction. LVEF (\%) was calculated as $[100 \times($ volume in diastole - volume in systole)/volume in diastole] by the modified Simpson's method. The parameter was averaged over 3 consecutive cycles by two independent observers.

Digital coronary angiography was conducted in surviving animals 3 days after infarction. A right or left femoral artery was surgically exposed and cut open with small sharp scissors. The guide wire $(0.97 \mathrm{~mm} \times 150$ $\mathrm{cm}$ ) was placed in the artery and a 6 French catheteriza- 
tion sheath was introduced and kept in place by a suture. Heparin $(1,250 \mathrm{U})$ was administered intra-arterially as a bolus. The left main coronary ostium was engaged, under fluoroscopy, using a 6 French JL 3.5 diagnostic catheter or 6 French XB 3.5 guiding catheter. Left coronary angiography, using iopamidol as the contrast medium, was performed in multiple views (right anterior oblique, anteroposterior, and left anterior oblique views). The complete occlusion of LAD was evaluated.

\section{Postmortem infarct size and ligation site}

The miniature pigs were euthanized with $10 \% 30 \mathrm{ml}$ potassium chloride i.v. to arrest the heart in diastole under deep anesthesia at 4 weeks after MI. The heart was excised to determine the whole length of LAD, the distance from the ligation point to the LAD ostium and the branching pattern of LAD. The percentile of LAD ligation $(\%)$ was calculated as $[100 \times$ (distance from the ligation point to the LAD ostium)/(the whole length of LAD)] (Fig. 1). Then, the heart was cut into 8-mm thick transverse slices in parallel to the atrioventricular groove to assess the infarct size. The infarct area that appeared pale was excised and calculated as the percentage of the ventricular mass. At the end of the experiment, sections from the peri-infarct area were stained with hematoxylineosin (HE) and Mallory solution.

\section{Statistical analysis}

Descriptive variables are presented as mean \pm standard deviation (SD) for measurement variables and percentages for numeration variables. Comparison between groups was performed with the $t$ or $t$ ' test for measurement data, and the Likelihood Ratio Chi-Square test for numeration data. Values of $P<0.05$ were considered statistically significant. The variation of each parameter was compared between the two groups using the homoscedasticity $F$ test and coefficient of variance $(C V)$. Linear regression analysis was used to analyze the correlation between the LAD ligation point and the infarct size or LVEF.

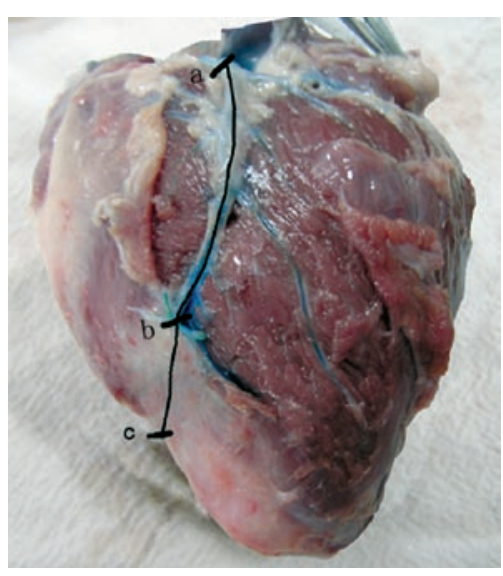

Fig. 1. Postmortem examination of LAD: the percentile of LAD ligation (\%) was calculated as $(\mathrm{ab} / \mathrm{ac} \times$ $100 \%)$, that is $[100 \times($ distance from ligation point to LAD ostium)/whole length of LAD]. a: LAD ostium; b: ligation point; c: turning point at apex.

\section{Results}

\section{Adverse events}

All 47 pigs developed ventricular premature contraction during surgery, and 14 of them experienced ventricular fibrillation, which was blocked by direct current defibrillation successfully in 6 pigs, but unsuccessfully in the other 8 pigs. Thus, 39 of the 47 pigs survived the surgical intervention. All ventricular arrhythmia occurred within the first 35 min after LAD occlusion.

As shown in Table 1, there were no significant differences in the incidences of ventricular fibrillation, death, wound disruption and infection between groups A and B.

\section{LVEF and its variation}

There was no significant difference in baseline (preMI) LVEF between groups A and B, while post-MI LVEF decreased to a greater extent in group A than in group $B$ (Table 2).

$\mathrm{CV}$ of post-MI LVEF in group B was smaller than that in group A. Furthermore, there existed heterogeneity of variance in post-MI LVEF between the two groups (Table 2). These results suggest that the left ventricular function in group $\mathrm{B}$, created by ligating LAD with its 
Table 1. Adverse events

\begin{tabular}{lrrc}
\hline & Group A & Group B & $P$ value \\
\hline Ventricular fibrillation & $37.50 \%(9 / 24)$ & $21.74 \%(5 / 23)$ & 0.235 \\
Death & $20.83 \%(5 / 24)$ & $13.04 \%(3 / 23)$ & 0.475 \\
Wound disruption & $0.00 \%(0 / 19)$ & $5.00 \%(1 / 20)$ & 0.243 \\
Wound infection & $5.26 \%(1 / 19)$ & $10.00 \%(2 / 20)$ & 0.575 \\
\hline
\end{tabular}

Percent figures are animal number suffering from events/total animal number.

Table 2. Variation of LVEF in the two groups

\begin{tabular}{lccc}
\hline & Group A $(\mathrm{n}=19)$ & Group B $(\mathrm{n}=20)$ & $P$ value \\
\hline LVEF pre-MI & & & \\
$\quad$ Value (\%) & $67.94 \pm 6.50$ & $67.42 \pm 5.39$ & 0.786 \\
$C V$ & 9.56 & 8.00 & \\
Variance & 42.22 & 29.07 & 0.294 \\
LVEF post-MI & & & \\
Value (\%) & $36.16 \pm 8.92$ & $46.52 \pm 5.00$ & $<0.001$ \\
$C V$ & 24.68 & 10.75 & \\
Variance & 79.65 & 25.00 & 0.024 \\
Decreased LVEF post-MI & & & \\
Value (\%) & $31.78 \pm 11.34$ & $20.91 \pm 5.84$ & 0.001 \\
$C V$ & 35.68 & 27.94 & \\
Variance & 128.53 & 34.12 & 0.007 \\
\hline
\end{tabular}

Values are mean $\pm \mathrm{SD}$; Variance comparison was made by the homoscedasticity $F$ test; LVEF, left ventricular ejection fraction; MI, myocardial infarction; $C V$, coefficient of variation.

whole length as a reference, was less variable than in group A.

Position of LAD ligation, infarct size, and their variations

Postmortem examination showed that the site of LAD ligation was lower and the infarct size was smaller in group B than in group A (Table 3). Importantly, the distance from the ligation point to the LAD ostium and the ischemic size in group B varied less than in group A. In addition, there existed heterogeneity of variance in the percentile of LAD ligation and ischemic size between the two groups (Table 3, Fig. 2). These findings indicate that there was a more uniform ischemic size and LVEF in group B than in group A.

\section{Coronary branching patterns (angiography and} postmortem examination)

To elucidate the great variations in LVEF, position of LAD Ligation, and infarct size in group A, coronary branching patterns were analyzed by angiography and postmortem examination. Coronary angiography showed that all occlusion sections were visible in all 39 surviving pigs, suggesting that the surgery was successful. Great variations were noticed in the position, number and diameter of ventricular branches in the pigs (Fig. 2), indicating that it would be difficult to find the correct ventricular branches during thoracotomy. It may explain the great variations in infarct area and LVEF in group $\mathrm{A}$ which usd the diagonal branches as coordinates for ligation. Occasionally, vessels distal to the occlusion site were visible due to the existence of collateral circulation (one pig in group A and 2 pigs in group B), contributing to the survival of cardiomyocytes in the infarct areas (Fig. 3), however, we could not perform a statistical analysis because of the small sample size.

Pig postmortem examination showed that the left main coronary artery was $0-3 \mathrm{~mm}$ in length, which corresponded with the angiographic imaging (Figs. 1A-D, $2 \mathrm{~A}, 2 \mathrm{~B}, 3 \mathrm{C}$, and $3 \mathrm{D}$ ). The length of $\mathrm{LAD}$, which had 3 
Table 3. Variation of LAD ligation site and infarct size in the two groups

\begin{tabular}{lccc}
\hline & Group A (n=19) & Group B (n=20) & $P$ value \\
\hline Whole length of LAD & & & \\
Value $(\mathrm{cm})$ & $9.08 \pm 1.01$ & $8.74 \pm 0.92$ & 0.274 \\
$C V$ & 11.11 & 10.58 & \\
Variance & 1.02 & 0.86 & 0.805 \\
Distance from ligation point to LAD ostium & & & \\
Value (cm) & $4.00 \pm 1.12$ & $5.78 \pm 0.68$ & $<0.001$ \\
$C V$ & 27.99 & 11.76 & \\
Variance & 1.25 & 0.46 & 0.013 \\
Percentile of LAD ligation & & & \\
Value $(\%)^{\mathrm{a})}$ & $43.96 \pm 10.41$ & $66.22 \pm 4.73$ & $<0.001$ \\
$C V$ & 23.69 & 7.14 & \\
Variance & 0.01 & 0.00 & 0.002 \\
Infarct size & & & \\
Value $(\%)^{\mathrm{b})}$ & $23.75 \pm 7.94$ & $12.79 \pm 2.69$ & $<0.001$ \\
$C V$ & 33.43 & 21.04 & \\
Variance & 63.01 & 13.62 & 0.001 \\
\hline
\end{tabular}

${ }^{a}$ Calculated as $[100 \times($ distance from ligation point to LAD ostium $) /($ whole length of LAD $)]$;

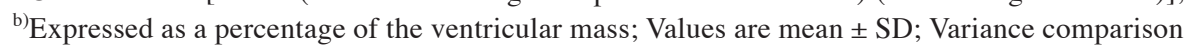
was made by the homoscedasticity $F$ test; LAD, left anterior descending artery; $C V$, coefficient of variation.
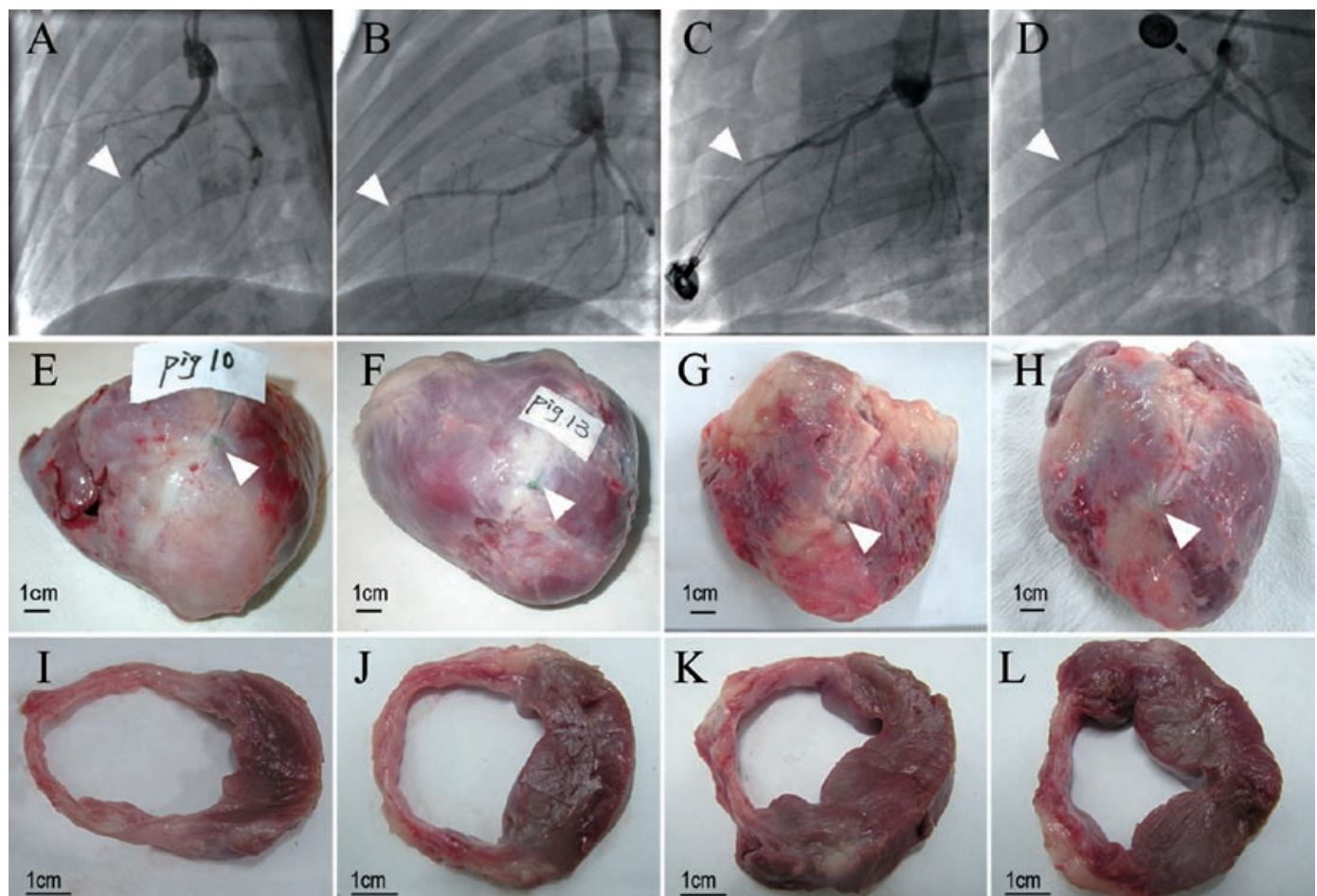

Fig. 2. Left coronary angiograms showing great variation in location, number and diameter of diagonal branches in both group A (A, B) and group B (C, D). A: tiny and rare diagonal branches invisible in open-chest operation; B: high position of the first diagonal branch as well as the existence of a fat pad, resulting in misjudgment in open-chest surgery. Variations of diagonal branches in group B (C, D) are also ostensible; however, their influence on the ligation site was minimal. E-H: corresponding ventricular gross appearance. I-L: corresponding cross-sectional specimen below papillary muscle level. Pathological examination showing the variable infarct location and size in group A (E and F; I and J) which were reproducible in group $\mathrm{B}(\mathrm{G}$ and $\mathrm{H} ; \mathrm{K}$ and $\mathrm{L})$. White arrowheads designate the ligation points of LAD. 


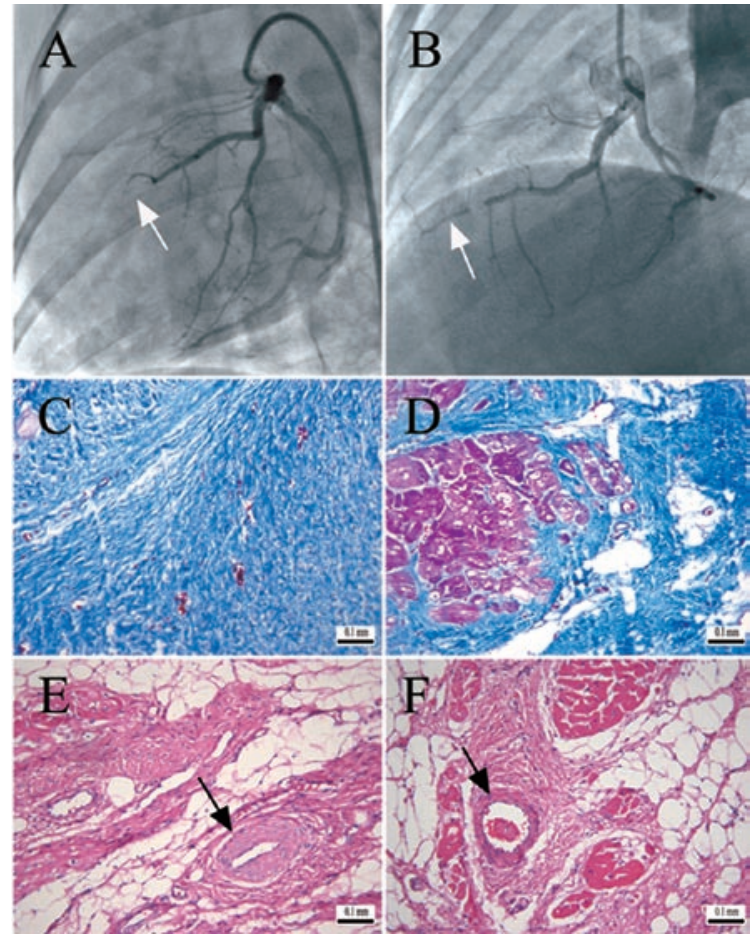

Fig. 3. Coronary angiograms showing LAD ligation without (A) and with collateral vessels (B). At 4 weeks after MI, a large scar area (C, Mallory stain) was formed with vascular obliteration (E, HE stain) in an animal without collateral vessels. In contrast, islands of surviving cardiomyocytes (D, HE stain) and patent vessels (F, HE stain) were present in animals with collateral vessels.

to 6 visible LV branches, from the base to the apex averaged $(8.78 \pm 0.25) \mathrm{cm}$. The extra-pericardium fat pad was thick in the atrioventricular groove and extended along the ventricular groove, covering the first half of LAD in all animals. The myocardial bridge was seen crossing an LAD segment anatomically in 7 pigs ( 3 in group A and 4 in group B) and angiographically in 3 pigs (one pig in group A and 2 pigs in group B) (Fig. 4D and $4 \mathrm{E})$. In addition, the LAD branches penetrated the myocardial layer at the very beginning in all 47 animals (Fig. 4B and $4 \mathrm{C}$ ). These fat pads and myocardial bridges made it difficult to view the LAD branches during the surgical procedure. After removal of the tectorial tissue, the underlying ventricular branches were not accompanied uniformly by the corresponding vein (Fig. 4A-C). The branching patterns of the LAD tree were irregular, which was consistent with the coronary angiographic findings.

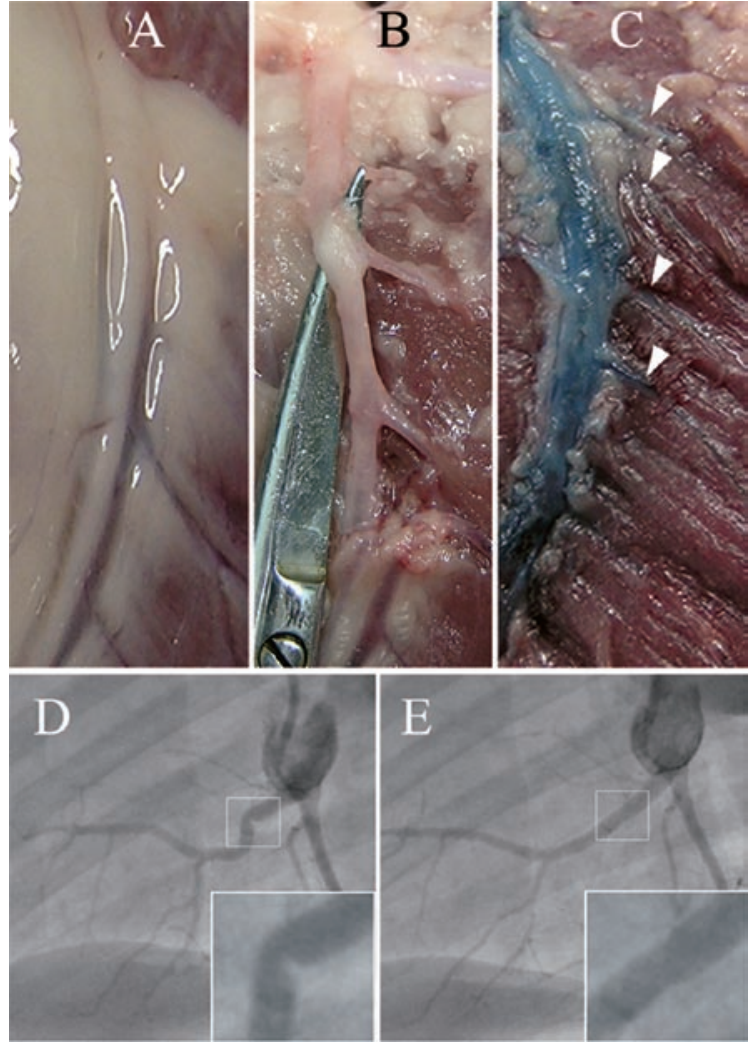

Fig. 4. Invisibility of ventricular branches. A: the fat pad along the atrioventricular groove was obvious, and the superficially dark red vessel branches are veins not arteries. B: a corresponding view after removal of the tectorial tissue. The underlying ventricular branches are unearthed, and not accompanied uniformly by the corresponding vein. $\mathrm{C}$ : ventricular branches penetrating into the myocardium from the very beginning (arrowhead), which is universal in pigs. D and E: coronary angiograms showing the "milking phenomenon" of the myocardial bridge at the proximal segment of LAD, characterized by narrowing during the systolic period (D) and recovery during the diastolic period (E).

The $\mathrm{D}_{1}$ and $\mathrm{D}_{2}$ branches arose from the $18 \pm 5 \%$ and 34 $\pm 8 \%$ section of LAD, respectively.

\section{Correlation of the LAD ligation point and infarct size or ventricular function}

To further identify the relations of occlusion location with ischemic size and ventricular function, correlation and regression analyse were performed. The correlation between the percentile of LAD ligation (x) and infarct size or ventricular function (y) was: $y=-0.54 x+48.06$, 

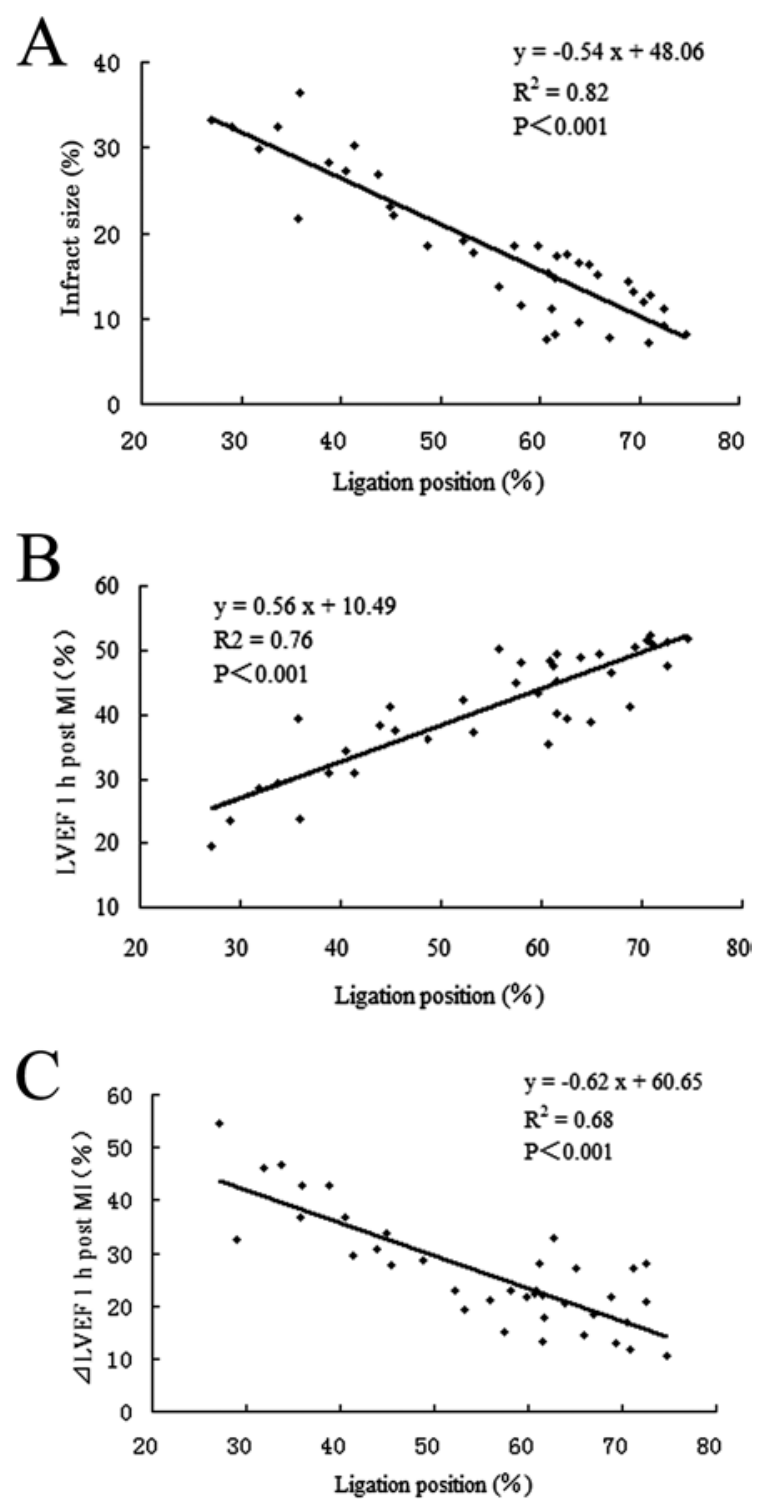

Fig. 5. Linear regression between percentile of LAD ligation and infarct size, ventricular function. A: infarct size; B: post-MI LVEF; C: decrease in post-MI LVEF; LVEF, left ventricular ejection fraction; $\triangle \mathrm{LVEF}$, decrease in left ventricular ejection fraction; MI, myocardial infarction.

$\mathrm{r}=0.90(P<0.001)$ for infarct size $(\%) ; \mathrm{y}=0.56 \mathrm{x}+10.49$, $\mathrm{r}=0.87(P<0.001)$ for LVEF post-MI $(\%) ; \mathrm{y}=-0.62 \mathrm{x}+$ $60.65, \mathrm{r}=0.82(P<0.001)$ for decreased LVEF post-MI (Fig. 5). According to the regression equations, we infer that LVEF would be 50, 45, and $30 \%$ corresponding to 70,60 , and $35 \%$ of LAD ligation, respectively.

\section{Discussion}

Our study is the first to reveal that the variation of ligation point of $\mathrm{LAD}$, ischemic size and LVEF after MI were greater in miniature pigs when diagonal branches were used as ligation coordinates than when LAD length was used as a reference, indicating that using LAD length as a reference produces a more stable and reliable MI model in pigs. This finding is of great significance in animal studies of heart failure, coronary artery disease, and stem cell therapy. For instance, intracoronary autologous bone-marrow cell transfer after acute MI can improve LVEF as little as 5\% [20]. Under such circumstances, a stable MI model is imperative to minimize the possible confounding effect $[1,17]$.

Conventionally, ventricular branches, rather than LAD itself, have been predominantly used as the occlusion reference in establishing open-chest MI models of pigs. It is commenly thought that by occluding LAD distal to $D_{1}, D_{2}$, or $D_{3}$ branch $[2,12,13]$, the size of the injured myocardium and the cardiac function can be well controlled by identification of these branches, which are supposed to be visible in surgery and less variable in anatomy. In fact, "blind" ligation is inevitable in many cases. First, ventricular branches feature great diversity in their location, number, and diameter, as observed in our and other studies of left coronary angiography and autopsy $[8,9]$. The irregular branching patterns of LAD make it difficult to identify which branch is the $D_{1}$ or $D_{2}$ branch. Secondly, tectorial tissues of the coronary artery including thick fat pads and the myocardial bridge [18], as well as limitation of the visual field in intercostal thoracotomy, may inhibit to some extent the precise judgment of these branches during surgical procedures. Thirdly, the distribution of superficial and visible coronary vein branches is not always in line with the branching pattern of $\mathrm{LAD}$, so they cannot serve as an indicator of artery branches. Indeed, we occasionally mistook the $\mathrm{D}_{1}$ or $\mathrm{D}_{3}$ branch for the expected $\mathrm{D}_{2}$ branch in the present study. These problems are attributable to the great variation in ligation point, infarct size and cardiac function in MI models.

Conversely, using the LAD length as the reference could standardize the occlusion site of LAD and avoid problem of branching variation and "blind" ligation, and 
establish a more stable and reliable MI model. Actually, our findings that the LAD length is superior to coordinates of ventricular branches is validated by the evidence that open-chest methods are more advantageous than closed-chest approaches, in procedures such as injection of thrombogenic material and occlusion by balloon catheter, due to a well-controlled occlusion site using the whole LAD length as a reference [10]. As the trunk of the left coronary artery in pigs is short (approximately $0-3 \mathrm{~mm}$ in this series), the initial point of LAD is easy to locate, and is approximately at the junction between the left atrium and the aortic root. As such, with full exposure of LAD in parasternal thoracotomy, it is feasible to define the percentile of LAD just by means of expanding a suture silk from the initial point of LAD to the apex of the heart.

The results of this study reconfirm the inverse relationship between the infarct size and LVEF. The larger the infarct scar, the lower the ejection fraction. More importantly, our study showed a good correlation between the percentile of the occlusion point and ventricular function, which could be used to determine the appropriate ligation site of LAD to satisfy different study purposes. If an experiment aims to establish a heart failure model, the ligation site should be shifted to a higher level. In this case, however, the risk of exposing animals to possible adverse outcomes (such as ventricular fibrillation and death) must be weighed against the benefit of significant decrease in left ventricular function. Pigs are susceptible to fibrillation [15], and will be in danger of dying if the ischemic area is over $25 \%$ of the left ventricle [8]. Therefore, the high death rate is a challenge, especially when a higher level of LAD ligation is adopted for constructing a heart failure model. In this study, LAD was occluded between the first and the third quartile of its length, which is in accordance with most experimental settings of acute MI and heart failure. It is noteworthy that the regression formulations drawn from our data should not be applied unlimitedly from the perspective of either statistics or pathology. That is, the change of cardiac function will definitely outreach the range of the prediction of the regression formulation when LAD is occluded at more than 75 percentile or less than 25 percentile of its length. Pigs rarely survive excessive ligation proximal to the $\mathrm{D}_{1}$ branch, which arose from the $18 \pm 5 \%$ section of LAD in this series. Notably, the same ligation percentile of LAD does not mean an identical decline of LVEF because of the individual variations in the predominance type of the coronary artery, the length of LAD, the collateral circulation (Fig. 3 ), the baseline cardiac function, and the branching pattern of LAD. It is a limitation of this study that we did not investigate cardiac biomarkers such as cardiac enzymes and brain natriuretic peptide (BNP). A combination of biomarkers and LVEF may be reflective of cardiac function in a more comprehensive way.

From the perspective of thoracotomy, the parasternal approach boasts its thorough exposure of LAD and intact pleura when compared with the intercostal pathway. On account of the maintenance of intrapleural negative pressure and no risk of open pneumothorax, we speculate that blood gas is normal during the procedure, even without mechanical ventilation. Despite this, in our study, mechanical ventilation was still maintained lest fibrillation should occur after acute MI, knowing that pigs are especially prone to the development of lethal ventricular arrhythmias [15]. Nevertherless, the parasternal approach has its own disadvantages. For instance, it is difficult to suture the sternum with the costal cartilage, and wound disruption is more likely to occur because the parasternal wound assumes greater pressure than the intercostal pathway. One pig in our study developed wound disruption. There was no significant difference in the incidence of wound infection and pericardial adhesion between the two approaches.

In summary, the pig MI model of ligating LAD distal to its ventricular branches is less satisfactory due to its greater variations in left ventricular function and ischemic area, as well as the problem of identifying nonuniform branches and their invisibility. Occluding LAD using its whole length as a reference rather than diagonal branches as coordinates may be advisable for establishing a reproducible and reliable MI model, since it enables standardization of the location of the occlusion and ischemic size.

\section{Acknowledgments}

This work was supported by the Shanghai Scientific Research Fund (06DJ14001), Program for Shanghai 
Outstanding Medical Academic Leader (LJ06008), National Basic Research Program of China (No. 2006CB943704).

\section{References}

1. Ahn, D., Cheng, L., Moon, C., Spurgeon, H., Lakatta, E.G., and Talan, M.I. 2004. Induction of myocardial infarcts of a predictable size and location by branch pattern probabilityassisted coronary ligation in C57BL/6 mice. Am.J. Physiol. Heart Circ. Physiol. 286: H1201-1207.

2. Campbell, C.D., Takanashi, Y., Laas, J., Meus, P., Pick, R., and Replogle, R.L. 1981. Effect of coronary artery reperfusion on infarct size in swine. J. Thorac. Cardiovasc. Surg. 81: 288-296.

3. Dick, A.J., Guttman, M.A., Raman, V.K., Peters, D.C., Pessanha, B.S., Hill, J.M., Smith, S., Scott, G., McVeigh, E.R., and Lederman, R.J. 2003. Magnetic resonance fluoroscopy allows targeted delivery of mesenchymal stem cells to infarct borders in Swine. Circulation 108: 28992904.

4. Faithfull, N.S., Erdmann, W., Fennema, M., and Kok, A. 1986. Effects of haemodilution with fluorocarbons or dextran on oxygen tensions in the acutely ischaemic myocardium. Br. J. Anaesth. 58: 1031-1040.

5. Fujiwara, H., Fujiwara, T., Tanaka, M., Onodera, T., Miyazaki, S., Wu, D.J., Matsuda, M., Sasayama, S., and Kawai, C. 1988. Detection of early myocardial infarction in formalin-fixed, paraffin-embedded tissue. Am.J. Cardiovasc. Pathol. 2: 57-61.

6. Fujiwara, H., Onodera, T., Tanaka, M., Miyazaki, S., Wu, D.J., Matsuda, M., Kawamura, A., Ishida, M., Takemura, G., and Fujiwara, Y. 1989. Acceleration of cell necrosis following reperfusion after ischemia in the pig heart without collateral circulation. Am. J. Cardiol. 63: 14-18E.

7. Galinanes, M., Garcia-Dorado, D., Eizaga, J., Solares, J., Riesgo, M., Fdez-Aviles, F., and Gomez Nebreda, M.J. 1987. Transient occlusion of the left anterior descending coronary artery in pigs. Eur. Surg. Res. 19: 246-253.

8. Kamimura, R., Suzuki, S., Nozaki, S., Sakamoto, H., Maruno, H., and Kawaida, H. 1996. Branching patterns in coronary artery and ischemic areas induced by coronary arterial occlusion in the CLAWN miniature pig. Exp. Anim. 45: 149-153.

9. Kassab, G.S., Berkley, J., and Fung, Y.C. 1997. Analysis of pig's coronary arterial blood flow with detailed anatomical data. Ann. Biomed. Eng. 25: 204-217.

10. Krombach, G.A., Kinzel, S., Mahnken, A.H., Gunther, R.W., and Buecker, A. 2005. Minimally invasive close-chest method for creating reperfused or occlusive myocardial infarction in swine. Invest. Radiol. 40: 14-18.

11. Lee, R., Nitta, T., Schmid, R.A., Schuessler, R.B., Harris, K.M., and Gay, W.A. Jr. 1998. Retrograde infusion of lidocaine or L-arginine before reperfusion reduces myocardial infarct size. Ann. Thorac. Surg. 65: 13531359.

12. Liu, J., Hu, Q., Wang, Z., Xu, C., Wang, X., Gong, G., Mansoor, A., Lee, J., Hou, M., Zeng, L., Zhang, J.R., Jerosch-Herold, M., Guo, T., Bache, R.J., and Zhang, J. 2004. Autologous stem cell transplantation for myocardial repair. Am. J. Physiol. Heart Circ. Physiol. 287: H501511.

13. Lutter, G., Yoshitake, M., Takahashi, N., Nitzsche, E., Martin, J., Sarai, K., Lutz, C., and Beyersdorf, F. 1998. Transmyocardial laser-revascularization: experimental studies on prolonged acute regional ischemia. Eur. J. Cardiothorac. Surg. 13: 694-701.

14. Perna, A.M., Masini, E., Nistri, S., Bani Sacchi, T., Bigazzi, M., and Bani, D. 2005. Human recombinant relaxin reduces heart injury and improves ventricular performance in a swine model of acute myocardial infarction. Ann. N.Y. Acad. Sci. 1041: 431-433.

15. Suzuki, M., Asano, H., Tanaka, H., and Usuda, S. 1999. Development and evaluation of a new canine myocardial infarction model using a closed-chest injection of thrombogenic material. Jpn. Circ. J. 63: 900-905.

16. Triulzi, M.O., Esposti, D., Mattioli, R., Signorini, G., Cirino, D., Colombo, D., Fumagalli, F., Aguggini, G., and Maggi, G.C. 1988. First seconds after acute experimental ischemic: changes in isovolumic contraction and relaxation. G. Ital. Cardiol. 18: 218-224.

17. van den Bos, E.J., Mees, B.M., de Waard, M.C., de Crom, R., and Duncker, D.J. 2005. A novel model of cryoinjuryinduced myocardial infarction in the mouse: a comparison with coronary artery ligation. Am. J. Physiol. Heart Circ. Physiol. 289: H1291-1300.

18. van Nie, C.J. and Vincent, J.G. 1989. Myocardial bridges in animals. Anat. Histol. Embryol. 18: 45-51.

19. Wayman, N.S., McDonald, M.C., Chatterjee, P.K., and Thiemermann, C. 2003. Models of coronary artery occlusion and reperfusion for the discovery of novel antiischemic and antiinflammatory drugs for the heart. Methods Mol. Biol. 225: 199-208.

20. Zhang, S., Sun, A., Ge, J., Yao, K., Huang, Z., Wang, K., and Zou, Y. 2009. Intracoronary autologous bone marrow stem cells transfer for patients with acute myocardial infarction: a meta-analysis of randomised controlled trials. Int. J. Cardiol. 136: 178-185. 